\title{
DNM1L, a key prognostic predictor for gastric adenocarcinoma, is involved in cell proliferation, invasion, and apoptosis
}

\author{
XIAO-WU XU ${ }^{1,2}$, XIAO-MIN YANG ${ }^{3}$, WEI-JIA ZHAO ${ }^{4}$, LEI ZHOU ${ }^{1}$, DE-CHUN LI $^{2}$ and YI-HU ZHENG ${ }^{4}$ \\ ${ }^{1}$ Department of General Surgery, The Second Affiliated Hospital and Children's Hospital of Wenzhou Medical University, \\ Wenzhou, Zhejiang 325027; ${ }^{2}$ Department of General Surgery, The First Affiliated Hospital of Soochow University, \\ Suzhou, Jiangsu 215006; ${ }^{3}$ Department of Pathology, Wenzhou People's Hospital, Wenzhou, Zhejiang 325000; \\ ${ }^{4}$ Department of General Surgery, The First Affiliated Hospital of Wenzhou Medical University, \\ Wenzhou, Zhejiang 325002, P.R. China
}

Received September 19, 2017; Accepted December 18, 2017

DOI: $10.3892 / \mathrm{ol} .2018 .9138$

\begin{abstract}
Dynamin-1-like protein (DNM1L) encodes a member of the dynamin superfamily of GTPases. It mediates mitochondrial and peroxisomal division and is involved in the regulation of apoptosis. However, its role in gastric cancer remains unclear. MKN-45 gastric cancer cells were transfected with short hairpin RNA (shRNA) to suppress DNM1L expression. MTT, flow cytometry, and Transwell assays were used to detect the changes in cell proliferation, apoptosis, and invasion, respectively. Immunohistochemistry was used to detect DNM1L expression in gastric adenocarcinoma specimens, and the association of DNM1L expression with clinicopathological features and prognosis was analyzed. After the suppression of endogenous DNM1L expression in MKN-45 cells with shRNA, cell proliferation and invasion rates were significantly reduced, whereas apoptosis was significantly increased (all $\mathrm{P}<0.01$ ). The expression of DNM1L was significantly higher in gastric adenocarcinoma specimens compared with that in pericarcinoma tissues $(\mathrm{P}<0.001)$. The expression of DNM1L increased with increasing infiltration depth, lymphatic metastasis, and higher tumor node metastasis stage $(\mathrm{P}<0.05)$. The expression of DNM1L associated negatively with prognosis $(\mathrm{P}<0.01)$. DNM1L plays a critical role in the proliferation, invasion and apoptosis of human gastric
\end{abstract}

Correspondence to: Dr Yi-Hu Zheng, Department of General Surgery, The First Affiliated Hospital of Wenzhou Medical University, Nan Bai Xiang Street, Ouhai, Wenzhou, Zhejiang 325002, P.R. China

E-mail: zhengyihu@foxmail.com

Dr De-Chun Li, Department of General Surgery, The First Affiliated Hospital of Soochow University, 188 Shizi Street, Suzhou, Jiangsu 215006, P.R. China

E-mail: lidechunsz@126.com

Key words: gastric cancer, DNM1L, proliferation, invasion, apoptosis, prognosis adenocarcinoma. DNM1L expression has prognostic significance for the survival of patients with gastric adenocarcinoma.

\section{Introduction}

Gastric cancer is the fifth most prevalent malignancy and the third leading cause of cancer-related death worldwide, and $70 \%$ of cases occur in east Asia (1,2), with China alone accounting for $42 \%$ of all newly diagnosed cases (3). However, the incidence of gastric cancer has decreased in most industrialized countries over the past three decades. The complete surgical removal of the tumor is an effective treatment for early gastric cancer, but at the time of diagnosis, the majority of gastric cancer patients are at an advanced stage of disease. The treatment of gastric cancer remains a challenge, and despite advances in surgical techniques, chemotherapy, and radiotherapy, the survival rate of gastric cancer patients remains unsatisfactory. The 5-year survival rate for advanced gastric cancer ranges from 25 to $35 \%(4,5)$, and this low rate is largely attributable to postoperative metastasis (6). There is still a large discrepancy between the success of treatment in mainland China and in Japan or Korea. Improvements in the early diagnosis and treatment of gastric cancer may continue to be the most effective strategy for improving patient survival. To improve the diagnosis and treatment of gastric cancer, an effective therapeutic target for this disease is required.

The mitochondrion is an organelle that is in a continuous state of dynamic fission-fusion equilibrium (7), which is important for maintaining normal cellular functions. If the equilibrium of the mitochondrion is disturbed, its energy-generating function is damaged and its DNA mutates. Mitochondrial dysfunction is closely related to tumor occurrence. The characteristics of tumor cells, such as their unlimited growth, resistance to apoptosis, evasion of immunological surveillance, invasion, and distant metastasis, are all related to mitochondrial dysfunction. Therefore, researchers have proposed new strategies that target the mitochondrion (8-10). A comprehensive understanding of the effects of mitochondria in the occurrence and development of tumors could provide theoretical guidance and new strategies for tumor therapies that target this organelle. Dynamin-1-like protein (DNM1L) belongs to the dynein GTP enzyme 
superfamily. DNM1L contains a unique proline-rich structural domain and three functional domains. The N-terminal domain has GTP enzyme activity; a dynein-like domain occurs in the middle region; and the C-terminal domain exerts a dynein-isogeny GTP enzyme effect $(11,12)$. DNM1L is a key regulatory factor mediating the dynamic fission-fusion equilibrium of the mitochondrion (13). It is usually found in cytosol, but after it is spliced, DNM1L is recruited by FIS1 in the mitochondrial outer membrane, and translocates from the cytosol to the mitochondrion, where it forms an oligomer. DNM1L then assumes a cyclical structure around the mitochondrion at the fission site, and ruptures the organelle by hydrolyzing GTP and extruding the mitochondrial membrane (14).

In this study, we determined the expression of DNM1L in patients with gastric cancer to gain a better understanding of DNM1L as a prognostic marker of this disease and the relationship between its expression and the clinicopathological features of the disease. We also examined the effects of DNM1L on the proliferation, apoptosis, and invasion capacity of gastric cancer MKN-45 cells. Our findings provide novel insights into the role of DNM1L in gastric cancer.

\section{Materials and methods}

Cells. Human gastric cancer cells SGC-7901 and AGS cells, and human embryonic kidney (293) cells were purchased from Shanghai Institutes for Biological Sciences (shanghai, China), and human gastric cancer MKN-45 cells were from the China Center for Type Culture Collection (Wuhan, China). All these cells were cultured in RPMI-1640 medium (Gibco BRL, Paisley, Scotland, UK) supplemented with $10 \%$ fetal calf serum (Sigma-Aldrich; Merck KGaA, Darmstadt, Germany).

Reagents. SYBR Master Mix was purchased from Takara Bio, Inc. (Otsu, Japan). The 3-(4,5-dimethylthiazol-2-yl)-2,5-diphenyltetrazolium (MTT) kit was obtained from Gen-View Scientific, Inc. (El Monte, FL, USA). The anti-DNM1L antibody (ab56788) was from Abcam (Cambridge, MA, USA).

Patients and preparation of tissue samples. In this study, we used a study cohort of 150 patients who underwent radical gastrotomy for gastric adenocarcinoma between January 2005 and December 2009 at The Second Affiliated Hospital of Wenzhou Medical University (Wenzhou, China) and were followed-up by December 2015. No patient was treated with radiotherapy or chemotherapy before surgery. The clinical information, including age, sex, tumor size, histological differentiation, recurrence, and metastasis, was obtained from the patients' medical records. The pathological TNM status was assessed according to the criteria of the TNM Classification of Malignant Tumors, American Joint Committee on Cancer (edition 7, 2010). This study was approved by the Ethics Committee of The Second Affiliated Hospital of Wenzhou Medical University, and the need for informed consent was waived. However, written informed consent for radical gastrotomy and the use of data for research purposes were obtained from patient prior to treatment.

Reverse transcription-quantitative polymerase chain reaction (RT-qPCR). Total RNA was prepared with TRIzol Reagent
(Pufei, Shanghai, China) and reverse transcribed with M-MLV reverse transcriptase (Promega Corporation, Madison, WI, USA). qPCR was performed with the SYBR Green Real-Time PCR Master Mix on the ABI Prism 7500 Sequence Detection System (Applied Biosystems; Thermo Fisher Scientific, Inc., Waltham, MA, USA). The primers used to amplify the DNM1L and glyceraldehyde 3-phosphate dehydrogenase (GAPDH) transcripts in the SYBR-Green assay were: DNM1L-F, 5'-GGTGAACCCGTGGATGATAAA-3' and DNM1L-R, 5'-CCTCAGGCACAAATAAAGCAG-3', which generated a 265-bp product; and GAPDH-F, 5'-TGACTTCAA CAGCGACACCCA-3' and GAPDH-R, 5'-CACCCTGTTGCT GTAGCCAAA-3'. The data were analyzed with the ABI 7500 System SDS software. The DNM1L mRNA expression levels were standardized to those of GAPDH mRNA by calculating $\Delta \mathrm{Cq}=\mathrm{Cq}(\mathrm{DNM} 1 \mathrm{~L})-\mathrm{Cq}(\mathrm{GAPDH})$. All experiments were performed in triplicate and repeated three times.

Lentiviral short hairpin RNA (shRNA) vector construction and transfection. A DNM1L-directed shRNA (sh-DNM1L: 5'-gcTACTTTACTCCAACTTATT-3') was designed based on the DNM1L gene sequence (accession no. NM_005690) and synthesized as follows: forward The oligonucleotides 5'-CcgggcTACTTTACTCCAACTTATTCT CGAGAATAAGTTGGAGTAAAGTAGCTTTTTg-3' and reverse 5'-aattcaaaagcTACTTTACTCCAACTTATTCT CGAGAATAAGTTGGAGTAAAGTAGC-3' were annealed and inserted downstream from the U6 promoter in the lentiviral vector GV248 (Jikai, Shanghai, China). A lentivirus carrying an shRNA-targeting nonsilencing sequence (5'-TTCTCC GAACGTGTCACGT-3') was used as the control (sh-Ctrl). The lentiviruses were generated by transfecting 293 cells with the GV248-sh-DNM1L plasmid, together with pHelper 1.0 and pHelper 2.0, with polyethylenimine (Sigma-Aldrich; Merck KGaA). For cell infection, the viral supernatants were supplemented with Polybrene (Santa Cruz Biotechnology, Inc., Dallas, TX, USA) and incubated with the cells for $8 \mathrm{~h}$. HepG2 cells were transfected with the lentiviral particles, and selected with puromycin $(2 \mu \mathrm{g} / \mathrm{ml})$ for 7 days. The cells stably expressing the shRNAs were confirmed with RT-qPCR.

Cell proliferation assay. Cells in the logarithmic growth phase were collected and digested with trypsin (Gibco; Thermo Fisher Scientific, Inc.). The cell suspension was prepared in culture medium at a density of $2 \times 10^{3}$ cells $/ \mathrm{ml}(100 \mu \mathrm{l})$ in a 96 -well plate, and the cells were incubated at $37^{\circ} \mathrm{C}$ under $5 \% \mathrm{CO}_{2}$. Cell proliferation was determined on days $1,2,3,4$, and 5 after seeding, with the MTT assay kit (Gen-View Scientific, Inc.), according to the manufacturer's instructions. Optical densities (ODs) were measured at $490 \mathrm{~nm}$ with a microplate reader (Infinite M1000; Tecan Group Ltd., Männedorf, Switzerland). Two independent experiments were performed.

Cell invasion assay. Culture medium (500 $\mu \mathrm{l})$ without fetal bovine serum was added to the upper and lower chambers of a Transwell apparatus and incubated at $37^{\circ} \mathrm{C}$ for $2 \mathrm{~h}$ to form a Matrigel matrix hydrate. After the Matrigel matrix was hydrated, the chambers were transferred into new wells. A cell invasion assay was performed in a 24-well Transwell chamber with $8 \mu \mathrm{m}$ pores (Corning Incorporated, Corning, 
NY, USA), according to the manufacturer's instructions. Cells were suspended in serum-free medium and seeded into Transwell inserts coated with growth-factor-reduced Matrigel (BD Biosciences, Franklin Lakes, NJ, USA). The bottom wells were filled with complete medium. After 24 h, Giemsa stain (Sigma-Aldrich; Merck KGaA) was added to the cells, and each chamber was photographed under an inverted fluorescence microscope (Olympus Corporation, Tokyo, Japan) for analysis. Nine random microscope fields (magnification, x200) were counted for each chamber and three independent experiments were performed.

Cell apoptosis assay. Cells were collected, seeded in 96-well plates, and allowed to grow to $80 \%$ confluence. The cells were then transferred to medium without serum or growth factors for 5 days. The apoptosis assay was performed with the Annexin V Apoptosis Detection kit APC (eBioscience; Thermo Fisher Scientific, Inc.), according to the manufacturer's protocol. The data were analyzed with the CellQuest software. Three independent experiments were performed.

Immunohistochemistry. Formalin-fixed paraffin-embedded tissue sections $(4 \mu \mathrm{m})$ were dewaxed, rehydrated in xylene and then in a $100-70 \%$ alcohol gradient, and washed in water. Antigen retrieval was performed with Antigen Unmasking Solution (Vectorlabs, Burlingame, CA, USA), according to manufacturer's instructions. The sections were blocked with $1 \%$ bovine serum albumin for $30 \mathrm{~min}$ at $24^{\circ} \mathrm{C}$, and were then incubated with a primary rabbit polyclonal anti-DNM1L antibody (diluted 1:100; Abcam, Cambridge, UK) for $45 \mathrm{~min}$ at $24^{\circ} \mathrm{C}$, followed by a secondary goat anti-rabbit immunoglobulin $\mathrm{G}(\operatorname{IgG})$ antibody for $30 \mathrm{~min}$ at $24^{\circ} \mathrm{C}$. The slides were washed three times with PBS. Positive staining was visualized with a DAB kit (Beijing Zhongshan Golden Bridge Biotechnology Co., Ltd., Beijing, China) for $5 \mathrm{~min}$ and then counterstained in hematoxylin. PBS was used instead of the primary antibody for the negative control. Immunostaining was blindly and semiquantitatively evaluated by two observers with no knowledge of the clinical data of the patients. DNM1L staining was scored for the percentage of positive cells and the intensity of staining in the cytoplasm. The scoring system for intensity was: 0 , no staining; 1 , weak staining; 2 , moderate staining; and 3, strong staining. The scoring system for the percentage of stained tumor cells was: $0,<5 \%$ stained cells; $1,5-25 \%$ stained cells; $2,26-50 \%$ stained cells; and 3, $>51 \%$ stained cells. A final score was the sum of the staining intensity and the percentage of stained cells: $0-1$, negative (-), 2-3, weakly positive (+); 4-6 positive (++); $>6$, strongly positive $(+++)$. A score of $0-1$ was considered DNM1L negative and a score $\geq$ was considered DNM1L positive.

Statistical analysis. The SPSS 17.0 software (SPSS, Inc., Chicago, IL, USA) was used for all statistical analyses. Measurement data were expressed as means \pm standard errors of the means (SEM). Multigroup comparisons of the means were performed using one-way analysis of variance with post hoc Student-Newman-Keuls test. Comparisons of count data were made with a $\chi^{2}$ test or Fisher's exact test. The Kaplan-Meier statistic was used to analyze survival, and the differences among groups were analyzed with the log-rank
A

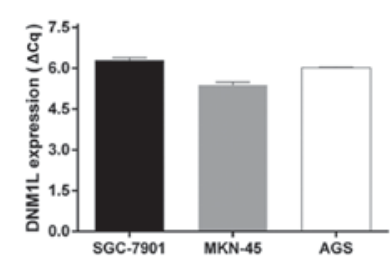

B

C

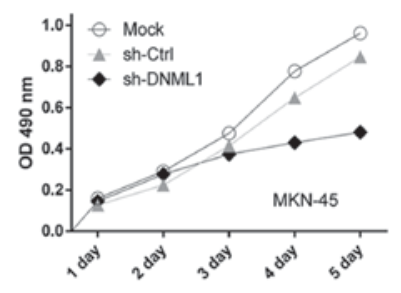

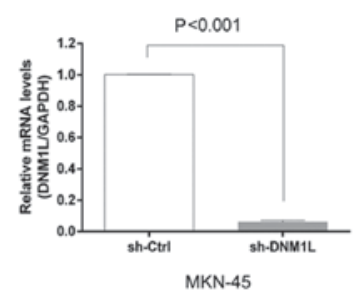

D

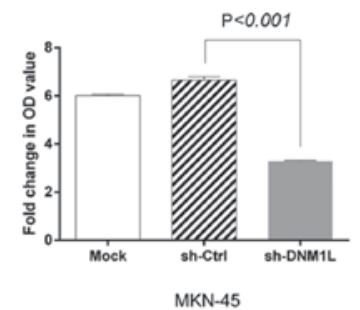

Figure 1. Expression of DNM1L and its effects on gastric carcinoma cell proliferation. (A) Expression of DNM1L mRNA in AGS, SGC-1901, and MKN-45 cells was determined with RT-qPCR. (B) Stable knockdown of DNM1L mRNA with shRNA in MKN-45 cells. (C) MKN-45 cells transfected with sh-DNM1L and sh-Ctrl were incubated for the indicated times. Proliferation was determined with an MTT assay. (D) Results of the MTT assay (optical density) of cells on day 5. Data are from three separate experiments and are presented as means \pm standard error of the mean. DNM1L, dynamin-1-like protein; shRNA, short hairpin RNA; Ctrl, control.
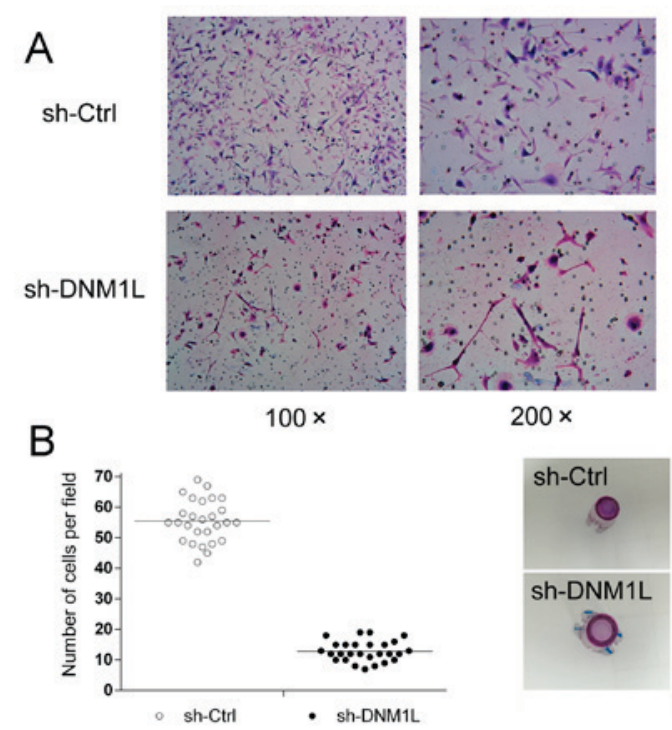

Figure 2. Effects of DNM1L on the invasive capacity of MKN-45 cells (A) Representative images under an inverted fluorescence microscope of the invasion assay of MKN-45 cells transfected with sh-DNM1L or sh-Ctrl (magnification, x100 or x200). (B) Numbers of invasive sh-DNM1L and sh-Ctrl cells per field were compared. Data are from three separate experiments and are presented as means \pm standard error of the mean. DNM1L, dynamin-1-like protein; Ctrl, control.

test. $\mathrm{P}<0.05$ was considered to indicate a statistically significant difference.

\section{Results}

DNMIL expression in gastric adenocarcinoma cells. We screened a panel of three gastric cancer cell lines (AGS, 

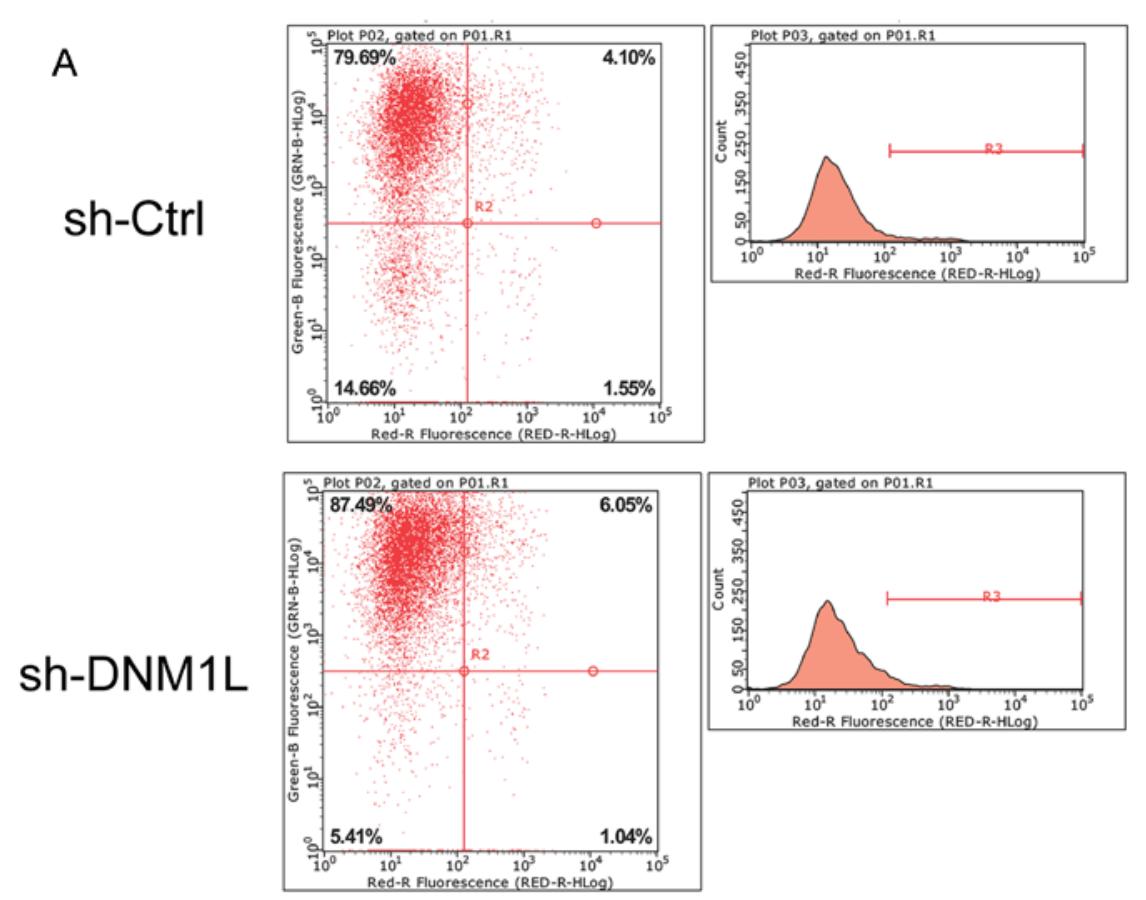

B

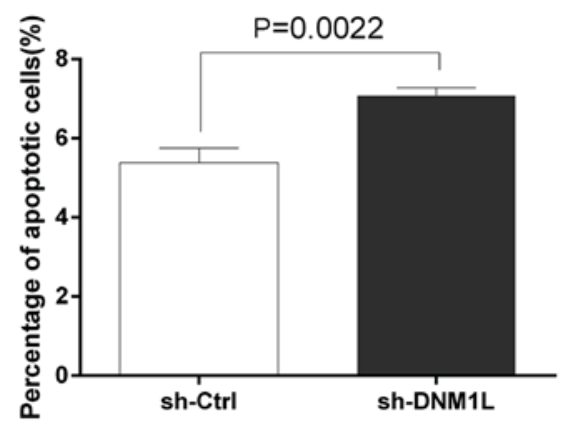

Figure 3. DNM1L suppresses apoptosis in MKN-45 cells. (A) Flow cytometry was used to detect and measure apoptotic cells. MKN-45 cells were infected with sh-DNM1L or sh-Ctrl, and cell apoptosis was detected with Annexin V-APC single staining and measured with flow cytometry. (B) Apoptosis rates of MKN45 cells transfected with sh-DNM1L or sh-Ctrl. Data are from three separate experiments and are presented as means \pm standard error of the mean. DNM1L, dynamin-1-like protein; Ctrl, control.

SGC-1901, and MKN-45) for DNM1L mRNA expression. High levels of DNM1L mRNA were observed in all three cell lines. The MKN-45 cells had the highest DNM1L mRNA expression levels and were selected for subsequent experiments (Fig. 1A). We engineered MKN-45-based cells with lentiviral transfection to express DNM1L shRNA. The level of DNM1L mRNA in the lenti-sh-DNM1L-transfected MKN-45 cells was examined with qPCR (Fig. 1B).

DNMIL accelerates MKN-45 cell proliferation. An MTT assay was used to assess cell viability as a marker of cell proliferation. MTT detected the proliferation of MKN-45 cells transfected with the sh-DNM1L lentiviral vector (sh-DNM1L) or the control vector (sh-Ctrl). The depletion of DNM1L significantly impaired the proliferation of MKN-45 cells (Fig. 1C). Moreover, the MTT OD value on day 5, normalized to that on day 1, was lower in the DNM1L-knockdown cells than in MKN-45 cells transfected with the control vector (Fig. 1D). This suggests that the depletion of DNM1L by shRNA in MKN-45 cells caused a dramatic reduction in cell proliferation.
DNM1L promotes the invasion of $M K N-45$ cells. A Transwell invasion assay was used to explore the effect of DNM1L on the invasion of MKN-45 cells. The number of DNM1L-knockdown cells in the high-power lens of each field of view was much lower than the number of MKN-45 cells transfected with the control vector (Fig. 2), suggesting that DNM1L promotes the invasion of $\mathrm{MKN}-45$ cells.

DNM1L inhibits the apoptosis of MKN-45 cells. The Annexin V Apoptosis Detection kit APC was used to investigate the effect of DNM1L on cell apoptosis. Flow cytometry showed that the apoptosis rate was higher in the DNM1L-knockdown cells than in the MKN-45 cells transfected with the control vector (Fig. 3). This suggests that DNM1L plays an important role in resistance to apoptosis.

Increased DNMIL expression is associated with a poor prognosis in gastric adenocarcinoma patients. We used immunohistochemistry to analyze the expression of the DNM1L protein in 150 primary gastric adenocarcinomas and the corresponding pericarcinoma tissues. Immunostaining 
Table I. Association of DNM1L expression with clinicopathological factors.

\begin{tabular}{|c|c|c|c|c|c|}
\hline \multirow[b]{2}{*}{ Clinicopathological parameters } & \multirow[b]{2}{*}{ No. } & \multicolumn{2}{|c|}{ DNM1L expression } & \multirow[b]{2}{*}{ Chi-square } & \multirow[b]{2}{*}{ P-value } \\
\hline & & Negative & Positive & & \\
\hline \multicolumn{6}{|l|}{ Age (years) } \\
\hline$<60$ & 63 & 12 & 51 & \multirow[t]{2}{*}{1.154} & \multirow[t]{2}{*}{0.283} \\
\hline$\geq 60$ & 87 & 11 & 76 & & \\
\hline \multicolumn{6}{|l|}{ Sex } \\
\hline Male & 105 & 17 & 88 & \multirow[t]{2}{*}{0.198} & \multirow[t]{2}{*}{0.656} \\
\hline Female & 45 & 6 & 39 & & \\
\hline \multicolumn{6}{|l|}{ Tumor diameter $(\mathrm{cm})$} \\
\hline$<5.0$ & 99 & 17 & 82 & \multirow[t]{2}{*}{0.758} & \multirow[t]{2}{*}{0.384} \\
\hline$\geq 5.0$ & 51 & 6 & 45 & & \\
\hline \multicolumn{6}{|l|}{ Tumor grade } \\
\hline Well & 25 & 6 & 19 & \multirow[t]{3}{*}{2.284} & \multirow[t]{3}{*}{0.319} \\
\hline Moderately & 70 & 11 & 59 & & \\
\hline Poorly & 55 & 6 & 49 & & \\
\hline \multicolumn{6}{|l|}{ Depth of invasion } \\
\hline $\mathrm{T} 1+\mathrm{T} 2$ & 56 & 13 & 43 & \multirow[t]{2}{*}{4.257} & \multirow[t]{2}{*}{0.039} \\
\hline $\mathrm{T} 3+\mathrm{T} 4$ & 94 & 10 & 84 & & \\
\hline \multicolumn{6}{|l|}{ TNM-stage } \\
\hline $\mathrm{I}+\mathrm{II}$ & 70 & 16 & 54 & \multirow[t]{2}{*}{5.723} & \multirow[t]{2}{*}{0.017} \\
\hline III+IV & 80 & 7 & 73 & & \\
\hline \multicolumn{6}{|l|}{ Lymph node metastasis } \\
\hline Negative & 60 & 16 & 44 & \multirow[t]{2}{*}{9.894} & \multirow[t]{2}{*}{0.002} \\
\hline Positive & 90 & 7 & 83 & & \\
\hline
\end{tabular}

TNM, tumor node metastatis; DNM1L, dynamin-1-like protein.

A

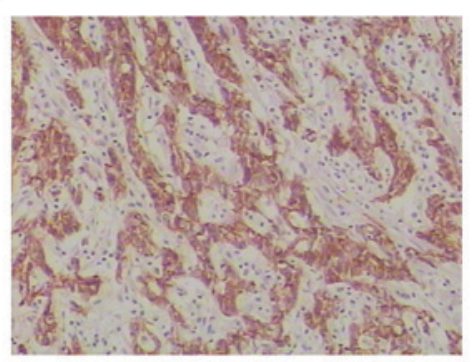

C

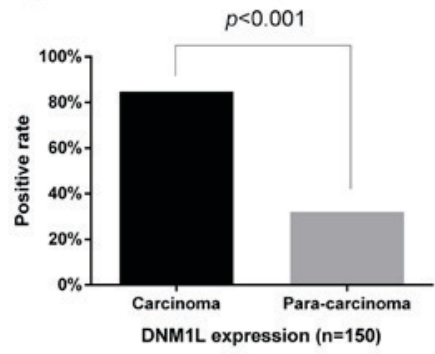

B

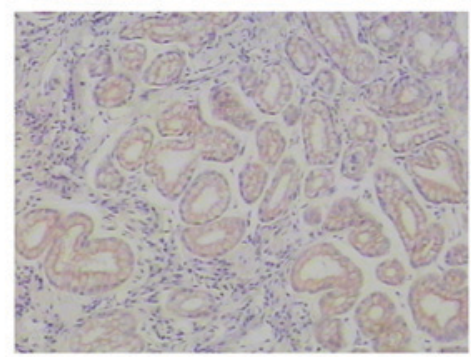

Overall Survival

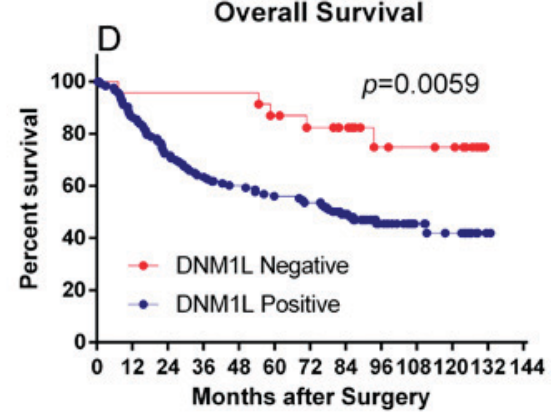

Figure 4. DNM1L confers poor prognosis in patients with gastric adenocarcinoma. (A) Immunohistochemical staining for DNM1L in carcinoma specimens (magnification, x200). (B) Immunohistochemical staining for DNM1L in pericarcinoma specimens (magnification, x200). (C) Frequency of DNM1L expression in carcinoma and pericarcinoma specimens ( $\mathrm{n}=150$ ), (D) Kaplan-Meier curves of the overall survival of patients negative or positive for DNM1L expression ( $\mathrm{P}=0.0059)$. DNM1L, dynamin-1-like protein. 
revealed that DNM1L expression was significantly higher in the carcinoma specimens $(84.67 \%)$ than that in the pericarcinoma specimens (32\%) (Fig. 4A-C). We also analyzed the associations between DNM1L expression and variables such as age, sex, tumor diameter, tumor grade, depth of invasion, TNM stage, and lymph-node metastasis. DNM1L expression associated significantly with the depth of invasion, lymph-node metastasis, and TNM stage, but not with the other factors tested (Table I). DNM1L-positive patients had a poorer survival outcome than DNM1L-negative patients [78 months (mean), 95\% confidence interval (CI): 68.6-87.4 vs. 113.5 months, 95\% CI: 99.4-127.7] (Fig. 4D).

\section{Discussion}

Mitochondria are highly dynamic organelles in living cells, and display continuous movement, fusion, and fission, forming the mitochondrial reticulum. The correct regulation of mitochondrial fission and fusion is essential for cellular homoeostasis. The balance between mitochondrial fission and fusion is controlled by a small cohort of mediators (OPA1, MFN1, and MFN2 for fusion and DNM1L for fission) (15). The organelle is closely associated with metabolism, development, and the death of cells (16). DNM1L is the key regulator mediating mitochondrial fission and fusion (16-18). It is primarily cytoplasmic, with a smaller fraction localizing to the outer mitochondrial membrane. The translocation of DNM1L from the cytoplasm to the mitochondrion appears to play a critical role in the regulation of mitochondrial fission (19). DNM1L has been implicated in neurodegenerative diseases $(20,21)$ and tumorigenesis of various kinds in humans. It is expressed at high levels in HCT116 and SW480 human colon cancer cells; the overexpression of DNM1L promotes the proliferation of colon cancer cells, and the downregulation of DNM1L reduces the proliferation and increases the apoptosis of these cells in vitro (22). Both immunohistochemistry and in vitro protein analyses showed that DNM1L expression is upregulated in oncocytic thyroid tumors, and the overexpression of DNM1L is associated with malignant oncocytic thyroid tumors. The inhibition of DNM1L activity also affects the migration and invasion capacities of thyroid cancer cells (23).

However, its role in gastric carcinoma remains unclear. Therefore, in this study, we investigated the expression and biological functions of DNM1L in gastric adenocarcinoma specimens and MKN-45 cells. Consistent with previous studies, we found that DNM1L was markedly upregulated in human gastric adenocarcinoma specimens relative to pericarcinoma specimens. DNM1L was also highly expressed in gastric carcinoma cell lines. DNM1L expression was associated with the depth of invasion, lymph-node metastasis, and TNM stage. Increased DNM1L expression was associated with a poor prognosis in gastric adenocarcinoma patients. Based on these data, DNM1L could be an attractive target for cancer therapy and warrants further exploration.

To study the effects of DNM1L knockdown on gastric carcinoma cells, a lentivirus-mediated shRNA approach was used to establish a cell line containing DNM1L-shRNA. An MTT assay, flow cytometry, and a Transwell assay were used to investigate the effects of DNM1L knock-down on cell proliferation, apoptosis, and migration/invasion, respectively. Our data revealed dramatically reduced MKN-45 cell proliferation and the induction of apoptosis after the transfection of an shRNA targeting DNM1L. We also found that the downregulation of DNM1L in MKN-45 cells reduced their invasive and migratory capacities. These results suggest that DNM1L is directly involved in the occurrence and invasiveness of gastric cancer.

In summary, our findings demonstrate, for the first time, that DNM1L expression is upregulated in gastric adenocarcinoma. It also correlates with lymphatic metastasis, infiltration depth, and the TNM stage, and could be a useful prognostic indicator of the survival of patients with gastric adenocarcinoma. The knockdown of DNM1L with shRNA potently reduced the proliferation and invasion of cells and induced their apoptosis in vitro. In future studies, we will increase the number of gastric cancer patients and include other pathological types of gastric cancer, besides adenocarcinoma, to investigate the role of DNM1L in gastric cancer. More gastric cancer cell lines should also be examined to determine the effects of DNM1L on cell proliferation, invasion, and other biological properties, both in vitro and in vivo.

\section{Acknowledgements}

The authors would like to thank Ms. Li-Wei Xie (Department of Pathology, The Second Affiliated Hospital and Children's Hospital of Wenzhou Medical University, Wenzhou, China) for her technical assistance in performing the immunohistochemical studies.

\section{Funding}

This study was supported by the Projects of Medical and Health Technology in Zhejiang Province (no. 2017KY453) and the Zhejiang Provincial Natural Science Foundation (no. LY18H160048).

\section{Availability of data and materials}

The datasets used and analyzed during the current study are available from the corresponding author on reasonable request.

\section{Authors' contributions}

XW and XY are researchers working in cancer biology and carried out the study. WZ and LZ undertook the statistical analysis. YZ along with DL designed the work and interpreted the results. YZ contributed to the writing of the manuscript. All the authors read and approved the final manuscript.

\section{Ethics approval and consent to participate}

This study was approved by the Ethics Committee of the Second Affiliated Hospital of Wenzhou Medical University, and the need for informed consent was waived. However, written informed consent for radical gastrotomy and the use of data for research purposes were obtained from patient prior to treatment. 


\section{Patient consent for publication}

As the approval for the use of data with written informed consent was obtained from patients and all identifying information was removed, the consent for publication was waived for this retrospective observational study.

\section{Competing interests}

The authors declare that there are no competing interests.

\section{References}

1. Torre LA, Bray F, Siegel RL, Ferlay J, Lortet-Tieulent J and Jemal A: Global cancer statistics, 2012. CA Cancer J Clin 65: 87-108, 2015

2. Ferlay J, Soerjomataram I, Dikshit R, Eser S, Mathers C, Rebelo M, Parkin DM, Forman D and Bray F: Cancer incidence and mortality worldwide: Sources, methods and major patterns in GLOBOCAN 2012. Int J Cancer 136: E359-E386, 2015.

3. Lin Y, Ueda J, Kikuchi S, Totsuka Y, Wei WQ, Qiao YL and Inoue $\mathrm{M}$ : Comparative epidemiology of gastric cancer between Japan and China. World J Gastroenterol 17: 4421-4428, 2011.

4. Waddell T, Chau I, Cunningham D, Gonzalez D, Okines AF, Okines C, Wotherspoon A, Saffery C, Middleton G, Wadsley $\mathrm{J}$, et al: Epirubicin, oxaliplatin and capecitabine with or without panitumumab for patients with previously untreated advanced oesophagogastric cancer (REAL3): A randomised, open-label phase 3 trial. Lancet Oncol 14: 481-489, 2013.

5. Smalley SR, Benedetti JK, Haller DG, Hundahl SA, Estes NC, Ajani JA, Gunderson LL, Goldman B, Martenson JA, Jessup JM, et al: Updated analysis of SWOG-directed intergroup study 0116: A phase III trial of adjuvant radiochemotherapy versus observation after curative gastric cancer resection. J Clin Oncol 30: 2327-2333, 2012.

6. Coburn NG, Lourenco LG, Rossi SE, Gunraj N, Mahar AL, Helyer LK, Law C, Rabeneck L and Paszat L: Management of gastric cancer in Ontario. J Surg Oncol 102: 54-63, 2010.

7. Roy M, Reddy PH, Iijima M and Sesaki H: Mitochondrial division and fusion in metabolism. Curr Opin Cell Biol 33: 111-118, 2015.

8. Zhao Y, Butler EB and Tan M: Targeting cellular metabolism to improve cancer therapeutics. Cell Death Dis 4: e532, 2013.

9. Boland ML, Chourasia AH and Macleod KF: Mitochondrial dysfunction in cancer. Front Oncol 3: 292, 2013.

10. Ubah OC and Wallace HM: Cancer therapy: Targeting mitochondria and other sub-cellular organelles. Curr Pharm Des 20: 201-222, 2014.
11. Bossy-Wetzel E, Barsoum MJ, Godzik A, Schwarzenbacher R and Lipton SA: Mitochondrial fission in apoptosis, neurodegeneration and aging. Curr Opin Cell Biol 15: 706-716, 2003.

12. Imai $\mathrm{Y}$ and Lu B: Mitochondrial dynamics and mitophagy in Parkinson's disease: Disordered cellular power plant becomes a big deal in a major movement disorder. Curr Opin Neurobiol 21: 935-941, 2011.

13. Chang CR and Blackstone C: Dynamic regulation of mitochondrial fission through modification of the dynamin-related protein Drp1. Ann N Y Acad Sci 1201: 34-39, 2010.

14. Cronin-Furman EN, Borland MK, Bergquist KE, Bennett JP Jr and Trimmer PA: Mitochondrial quality, dynamics and functional capacity in Parkinson's disease cybrid cell lines selected for Lewy body expression. Mol Neurodegener 8: 6, 2013.

15. Archer SL: Mitochondrial dynamics-mitochondrial fission and fusion in human diseases. N Engl J Med 369: 2236-2251, 2013.

16. Mishra P and Chan DC: Mitochondrial dynamics and inheritance during cell division, development and disease. Nat Rev Mol Cell Biol 15: 634-646, 2014.

17. Frank S, Gaume B, Bergmann-Leitner ES, Leitner WW, Robert EG, Catez F, Smith CL and Youle RJ: The role of dynamin-related protein 1, a mediator of mitochondrial fission, in apoptosis. Dev Cell 1: 515-525, 2001.

18. Tailor D, Hahm ER, Kale RK, Singh SV and Singh RP: Sodium butyrate induces DRP1-mediated mitochondrial fusion and apoptosis in human colorectal cancer cells. Mitochondrion 16: 55-64, 2014.

19. Park SJ, Park YJ, Shin JH, Kim ES, Hwang JJ, Jin DH, Kim JC and Cho DH: A receptor tyrosine kinase inhibitor, Tyrphostin A9 induces cancer cell death through Drpl dependent mitochondria fragmentation. Biochem Biophys Res Commun 408: 465-470,2011.

20. DuBoff B, Gotz J and Feany MB: Tau promotes neurodegeneration via DRP1 mislocalization in vivo. Neuron 75: 618-632, 2012

21. Yan J, Liu XH, Han MZ, Wang YM, Sun XL, Yu N, Li T, Su B and Chen ZY: Blockage of GSK3beta-mediated Drp1 phosphorylation provides neuroprotection in neuronal and mouse models of Alzheimer's disease. Neurobiol Aging 36: 211-227, 2015.

22. Inoue-Yamauchi A and Oda H: Depletion of mitochondrial fission factor DRP1 causes increased apoptosis in human coloncancercells. BiochemBiophys Res Commun421:81-85,2012.

23. Ferreira-da-Silva A, Valacca C, Rios E, Populo H, Soares P, Sobrinho-Simões M, Scorrano L, Maximo V and Campello S: Mitochondrial dynamics protein Drpl is overexpressed in oncocytic thyroid tumors and regulates cancer cell migration. PLoS One 10: $\mathrm{e} 0122308,2015$.

c) (7) $\odot$ This work is licensed under a Creative Commons Attribution-NonCommercial-NoDerivatives 4.0 International (CC BY-NC-ND 4.0) License. 